\title{
Positioning Parenting Differently: The Need for Southern Perspectives
}

\author{
*Nicola M. Cilliers. http://orcid.org/0000-0003-1946-54I7 \\ BSc. Occupational Therapy (UCT). Private practitioner, Cape Town, South Africa.
}

Despite the importance of the caregiver role in children's development and the reliance on caregivers in the therapy process, parenting has enjoyed limited attention in Occupational Therapy research. While it is gradually gaining more attention and being appreciated for its complexity, little is known about how parenting unfolds in the margins. Dominant parenting norms promoted globally are largely Eurocentric' and the resultant white, middle class positioning of professional discourse may offer a limited perspective on parenting.

This may sustain incoherence between how parenting unfolds in context and the therapeutic understanding of, and response to, families' needs. This opinion piece aims to promote the importance of appreciating and exploring southern parenting perspectives for meaningful work with families.

Emerging southern epistemologies reveal several relational and contextual complexities, situated within persisting structural inequalities, that characterise everyday life for many families. Along with cultural and intergenerational influences to be navigated in context, these factors create a cumulatively challenging parenting experience and shape families' subsequent occupational engagement. These insights not only demand the broadening of the parenting knowledge base but may hold implications for how parenting is understood as an occupation in the margins. Appreciating and pursuing plurality in parenting perspectives is essential to facilitate knowledge generation that speaks to those routinely excluded from the theorising process and to inform more responsive intervention.

Keywords: parenting, human occupation, southern epistemologies, human relations, structural inequality.

\section{INTRODUCTION}

Despite the importance of the caregiver role in children's development and the reliance on caregivers in the therapeutic process, parenting has enjoyed limited attention in occupational therapy research. This is especially the case for how parenting unfolds for families in the margins, or those on the periphery of dominant society. While research frequently focuses on caregivers' possession of professional knowledge, such as developmental milestones and positive parenting practices $^{2}$, little research appears to explore the indigenous and experiential knowledge of caregivers in the margins ${ }^{\prime}$ that may hold value for meaningful intervention. This is concerning given that interventions with families must be culturally and contextually relevant to be sufficiently supportive ${ }^{3}$, and thus demands the consideration and inclusion of multiple parenting perspectives.

This opinion piece aims to demonstrate the need for greater appreciation and exploration of southern parenting perspectives to further meaningful work with families. Dominant parenting perspectives informing occupational therapy practice will be described and contrasted with emerging literature that reveal the relational, contextual, and structural complexities of parenting in the margins. Implications for research and practice are briefly discussed.

\section{A brief overview of current parenting perspectives}

Parenting is traditionally considered an occupation performed by parents or other caregivers of children, but has recently been explored from a transactional perspective, acknowledging that both caregivers and children influence one another in the context of their unique situations. It has been argued that caregivers and children, in the performance of parenting, may engage together in co-occupations, such as play or feeding ${ }^{4}$ or family occupations ${ }^{5}$.
Sethi ${ }^{6}$ explored 'mothering' and particularly everyday parental decision-making in Virginia, USA. She described mothering as a relational role, shaped by family values and socio-cultural influences, and argued that, given the many occupations that form part of mothering (such as nurturing, practical caregiving and educating children), parenting should be viewed not as an occupation or cooccupation, but rather a complex role comprising many occupations. This, she argued, would avoid an individualistic perspective in which people are considered separate from their environment, culture, and past experiences ${ }^{6}$.

Due to the significant role they play in child health and development, caregivers are increasingly included as a meaningful resource for achieving timely and effective therapy progress. Parenting itself may become the focus of intervention, such as when children display behavioural challenges. These caregiver interventions aim to improve behaviour management ${ }^{7}$ through advice around routine and consistency ${ }^{8}$, how caregivers' behaviours influence children ${ }^{7}$ and positive behaviour-modification strategies ${ }^{8}$. Providing these interventions in ways that are relevant and appropriate for families requires an understanding of how parenting unfolds in context.

\section{The limitations of current perspectives}

Relational complexities shaping parenting

While current parenting perspectives hold value, the positioning of such knowledge must be considered in evaluating its relevance and appropriateness for families in the margins. Occupational therapy theorising has been situated largely within the academic environment, drawing from and upholding Western knowledge and culture?. The generally Eurocentric nature of parenting norms, represented in research and advocated for in practice, may therefore be inap- 
propriate for other cultures and contexts ${ }^{2}$.

A problematic feature of most parenting literature, for example, is their conceptualisation of parenting as occurring between parents (often mothers) and their children. This contrasts strongly with the complex household structures that are common in the margins. In South Africa for example, only $50 \%$ of children live with their biological parents ${ }^{10}$. In addition, caregivers from an isi-Xhosa community shared that parenting unfolds as a collective responsibility adopted by multiple caregivers. This practice is due to complex family dynamics in context, children spending substantial periods away from their immediate caregivers, as well as the mutual support offered amongst community members in raising one another's children. Considering this, they felt that strengthening social support mechanisms in communities was an essential part of supporting parenting ${ }^{3}$.

This collective approach to parenting suggests that southern parenting experiences could differ significantly from the parenting and mothering described in literature from Western Europe and the USA, where one or two parents are predominantly responsible for the children in the household. The interdependent nature of parenting corresponds with emerging southern epistemologies about human occupation; Ramugondo and Kronenberg " draw on the African ethic of Ubuntu to appreciate human interconnectedness in context, arguing that individuals are continuously influenced by what they are able or unable to do within groups, communities and broader society (i.e., by the wellbeing of the collective). Within this, human relations become particularly significant as shaping and being shaped by people's everyday occupational engagement (e.g., parenting) in context. Through relational agency, namely "human subjects' capacity to act upon, influence, or empower one another" 12:158, families may, within their structural realities, influence their collective wellbeing. The relational aspect of parenting, already recognised in some parenting literature ${ }^{6}$ and observed through everyday practices such as older children caring for younger family members, may thus warrant greater exploration as a potentially significant facet of parenting in the margins.

\section{Contextual and structural complexities shaping parenting}

The potential significance of human relations in families' occupational engagement and subsequent wellbeing may be especially pertinent given the many contextual challenges that characterise parenting in the margins. Factors such as poverty, parental mental health and family illness such as HIV/AIDS prevalence may shape parenting practices ${ }^{13}$. Poverty in particular impacts on caregivers' ability to provide physical resources for their children and places strain on caregivers' mental and physical health ${ }^{10}$ through the time and capacity needed to meet basic needs, such as food provision. In South Africa, it has been found that parenting is also influenced by the stress associated with keeping children safe in their communities; along with the intergenerational transmission of parenting practices, these challenges often informed harsher parenting practices and less time spent playing with their children ${ }^{3}$. Due to the prevalence of HIV/AIDS in many families, caregivers also felt burdened by the need to communicate with their children in an age-appropriate manner about HIV, how to use antiretroviral treatment and managing associated stigma ${ }^{3}$.

The socioeconomic challenges faced by many families are situated within, and are intimately related to, broader socio-historical contexts of discrimination and persisting structural inequalities relating to families' intersectional positioning. Cannon et al. ' argue, for example, that African American mothers may experience the intersection of their black, female and parent identities as creating a particular combination of everyday constraints shaping their parenting and subsequent judgement by others. The historical and socio-political context of systemic violence and structural inequalities to which their identities are subjected, seen in the USA through structures such as the policing system', creates an urgency to raise children who will survive a harsh world in which there is little room for error. Therefore, while African American mothers' 'violent' parenting practices are criticised in relation to dominant parenting norms, mothers described these practices as preparing their children for discriminatory contexts that may treat them unfairly in future'.

This reflects the occupational science construct of occupational choice, in which people's actions are guided by their "practical consciousness" 14:47 of what is perceived as possible and necessary within their contextual constraints. Families may have to manage everyday contextual stressors within macro-level structures, while simultaneously navigating the subsequent opportunities and demands inherent in their everyday parenting interactions with children. This is in addition to the intergenerational and cultural factors shaping parenting beliefs ${ }^{6}$ and how these may find expression in context; Asian caregivers in the United Kingdom, for example, shared the challenge of balancing their cultural expectations with contextual norms for parenting. Caregivers struggled to support their children's assimilation into the Western context while upholding their Eastern heritage, leading to conflict with their children who felt unduly pressured by their caregivers' expectations ${ }^{7}$.

The above positions parenting firmly within the structure-agency continuum, which appreciates occupations as both embodied in the person (agency) and embedded in context (structure) $)^{12}$.

\section{Implications for parenting research and practice}

The intricate ways in which structure and agency intersect in these parenting experiences - in addition to shaping families' needs -may hold implications for understanding how parenting unfolds as an occupation in the margins. The parenting approaches adopted by families as an apparent response to pervasive systemic forces, for example, may reflect a particular intentionality behind parenting to contest and/or mediate the effects of structure on the wellbeing of their children and families. In this way, rather than a role adopted by individual caregivers, parenting could be framed as a collective occupation for the ways in which it is enacted by caregivers, families and communities to influence "social cohesion or dysfunction, and/or the advancement of or aversion to a common good" "1:8. While this is one possible interpretation that would require further investigation, emerging southern literature certainly suggests an incomplete understanding of parenting, particularly as it unfolds in the margins, and the subsequent need to broaden the parenting knowledge-base for effective work with families.

Beyond meeting families' needs, understanding how parenting unfolds in context is especially significant given the potential of offered interventions to compromise parenting further. Dominant discourses defining 'good' parenting (and the structural inequalities encountered in pursuing them) may act as additional constraints for caregivers in their limited reflection of families' socio-historical experiences in context. It was found in a Canadian study, for example, that the often mother-centric nature of available support services served as one factor influencing the involvement of fathers within indigenous communities. This discourse intersected with their persistent economic exclusion and ongoing coloniality in context to restrict their capacity to care for their children ${ }^{15}$. 
A restricted understanding of parenting therefore not only limits appropriate intervention but may in this way perpetuate the very challenges that families need addressed. By continuing to draw mostly on Eurocentric parenting perspectives, therapists risk problematising families' alternate ways of doing, potentially upholding coloniality in the privileging of dominant parenting discourses and practices ${ }^{9}$. Appreciating plurality in parenting perspectives may facilitate knowledge generation that speaks to those routinely excluded from the theorising process and may shape greater partnership with families in the pursuit of health and wellbeing.

\section{CONCLUSION}

The white, middle class positioning of professional discourse may offer a limited perspective of parenting, potentially restricting the theory that informs occupational therapy intervention. Dominant perspectives of parenting may not fully encapsulate how parenting unfolds for families in the margins, leading to intervention approaches that are inappropriate and may cause families harm. Emerging southern literature describes relational, contextual, and systemic complexities that challenge parenting and may hold implications for how the profession understands parenting as an occupation. This demands the greater pursuit of southern epistemologies and the appreciation of plurality in parenting perspectives.

\section{REFERENCES}

I. Cannon C, Ferreira RJ, Buttell F. Critical race theory, parenting, and intimate partner violence: Analyzing race and gender. Research on Social Work Practice. 2019; 29(5): 590-602.

https://doi.org/10.1 177//049731518772151

2. September SJ, Rich E, Roman N. Association between knowledge of child development and parenting: A systematic review. Early Child Development and Care. 2017; 186(7): 1060-1078. https://doi.org/I0.2174/I87492240170901000।

3. Lachman JM, Sherr LT, Cluver L, Ward C, Hutchings J, Gardner F. Integrating evidence and context to develop a parenting programme for low-income families in South Africa. Journal of Child and Family Studies. 2016; 25: 2337-2352.

https://doi.org/10.1007/s 10826-016-0389-6

4. Price P, Stephenson SM. Learning to promote occupational development through co-occupation. Journal of Occupational Science. 2009; 16(3): 180-186.

https://doi.org/10.1080//442759I.2009.9686660

5. Bonsall A. "This is what we do": Constructing postmodern families through occupations. Journal of Occupational Science. 20 I 4; 2 I (3): 296-308. https://doi.org//0.1080/I442759I.2014.914459

6. Sethi C. Parental decision-making processes: Historical and sociocultural influences on everyday parenting occupations. [PhD thesis]. University of North Carolina. 2017 [cited 202I April 17]. Available from: file://C:/Users/27836/Downloads/Sethi_unc_0153D_I688I. pdf\%20(I).pdf

7. Bloomsfield L, Kendall S, Applin L, Attarzadeh V, Dearnley K, Edwards $L$, et al. A qualitative study exploring the experiences and views of mothers, health visitors and family support centre workers on the challenges and difficulties of parenting. Health and Social Care in the Community. 2005; 13(I): 46-55.

https://doi.org/10.1 I II/j.1365-2524.2005.00527.x

8. Beck AJ, Barnes KJ, Vogel KA, Oxford Grice K. The dilemma of psychosocial occupational therapy in public schools. Occupational Therapy in Mental Health. 2006; 22(I): I-I 7.

https://doi.org/I0.1300/j004v22n0I_OI
9. Ramugondo EL. Healing work: Intersections for decoloniality. World Federation of Occupational Therapists Bulletin. 20I8; 74(2): 83-9I. https://doi.org/10.1080/14473828.2018.152398।

10. Gould C, Ward C. Positive parenting in South Africa: why supporting families is key to development and violence prevention. Policy brief. 2015; 77: I-8.

https://www.saferspaces.org.za/uploads/files/PolBrief77.pdf

I I. Ramugondo EL, Kronenberg F. Explaining occupations from a human relations perspective: Bridging the individual-collective dichotomy. Journal of Occupational Science. 2013; 22(I): 3-16. https://doi.org/10.1080/14427591.2013.781920

12. Kronenberg F. Everyday enactments of humanity affirmations of post- 1994 Apartheid South Africa: A phronetic case study of being human as occupation and health. [PhD thesis]. University of Cape Town. 2018 [cited 2020 June 24]. Available from: https://open.uct.ac.za/handle/ I |427/2944 I

13. Sherr L, Macedo A, Cluver LD, Meinck F, Skeen S, Hensels IS, et al. Parenting, the oldest profession in the world - a cross-sectional study of parenting and child outcomes in South Africa and Malawi. Health Psychology and Behavioural Medicine. 2017; 5(I): I45- 165. https://doi.org/10.1080/21642850.2016.1276459

14. Galvaan R. The contextually situated nature of occupational choice: Marginalised young adolescents' experiences in South Africa. Journal of Occupational Science. 2015; 22(1): 39-53. https://doi.org/10.1080/14427591.2014.912124

15. Ball J. Fathering in the Shadows: Indigenous Fathers and Canada's Colonial Legacies. The ANNALS of the American Academy of Political and Social Science. 2009; 624(I): 29-48.

https://doi.org/10.1177/00027/6209334181

Corresponding Author

* Nicola M. Cilliers

Email: nicolamcilliers@gmail.com 